\title{
APREÇAMENTO DE DERIVATIVOS BIDIMENSIONAIS
}

\author{
Hugo Daniel de Oliveira Azevedo ${ }^{\S}$ \\ José Santiago Fajardo Barbachan ${ }^{\text {n }}$
}

\section{RESUMO}

Neste artigo analisamos o apreçamento de contratos que tenham seus resultados atrelados a mais de um ativo subjacente, em especial, opções bidimensionais. Para isto usamos a fórmula desenvolvida por Margrabe (1978) e o modelo de árvores de Rubinstein (1991a). Em seguida apresentamos exemplos práticos de opções bidimensionais e apreçamos estas opções. Além disto, sugerimos a incorporação de outros instrumentos, negociados no exterior, ao mercado de derivativos brasileiro.

Palavras-chave: apreçamento de derivativos, opções bidimensionais, opções de Margrabe.

\section{ABSTRACT}

In this article we analyze the pricing of contracts that have their payoffs linked to more than one underlying asset, in special, bidimensional options. To achieve this purpose we use the formula developed by Margrabe (1978) and the tree model of Rubinstein (1991a). Next, we present practical examples of bidimensional options and price these options. Moreover we suggest the incorporation of two other instruments, negotiated abroad, to the Brazilian derivative markets

Key words: derivative pricing, bidimensional options, Margrabe options.

JEL classification: G13. 


\section{INTRODUÇÃo}

Em seus trabalhos pioneiros, Black e Scholes (1973) e Merton (1973) encontraram uma fórmula fechada para o preço livre de arbitragem de uma opção vanilla européia num ativo subjacente. Porém, como é bem sabido, as hipóteses simplificadoras usadas são irrealistas, pois assumem que o mercado é perfeito, no sentido de que não existem fricções. Além disto, elas não capturam corretamente o comportamento dos retornos dos ativos financeiros. Desde então, várias extensões deste modelo têm sido desenvolvidas.

Uma extensão natural é considerar mais de um ativo subjacente. Esta extensão é muito importante, pois, como se sabe, os problemas multidimensionais são muito interessantes do ponto de vista acadêmico devido à sua complexidade matemática, bem como por suas aplicações práticas, porquanto muitas são as variáveis de mercado que afetam os contratos financeiros, tais como inflação, taxa de câmbio, taxa de juros etc.

No Brasil existem vários contratos com parâmetros atrelados à variação cambial, porque $o$ Brasil é um dos países que estão expostos a muita especulação cambial, daí a necessidade de se ter papéis bidimensionais com o propósito de hedging está justificada. Disto decorre a pergunta: como apreçar estes contratos?

Margrabe (1978) foi o primeiro a responder tal pergunta, obtendo uma solução analítica para o preço de uma opção européia de troca de um ativo por outro. Ele utilizou distribuições de probabilidade normais univariadas. Ademais, para o caso de opções americanas, ele demonstrou que tais opções valem mais "vivas" do que "mortas", ou seja, não existe um prêmio pelo exercício antecipado no caso em que os ativos subjacentes não pagam dividendos. Logo, a fórmula encontrada para opções européias seria também válida para opções americanas. Generalizações deste trabalho estão presentes em Stulz (1982) e Johnson (1987), enquanto que os trabalhos de Boyle (1988), Boyle, Evnine e Gibbs (1989) e Kamrad e Ritchken (1991) utilizaram a abordagem lattice para tratar o mesmo problema e seus similares.

Em seguida surgiu uma coleção de trabalhos de Rubinstein (1991-1994), dedicada ao estudo e avaliação destes tipos de instrumentos, e é em um destes textos que Rubinstein desenvolve um modelo binomial para a razão dos preços dos dois ativos subjacentes. Sua abordagem pode ser considerada um espelho do modelo de Cox, Ross e Rubinstein (1979).

Até este ponto, os modelos desenvolvidos se baseavam na hipótese de mercados completos, o que se traduzia em uma única medida martingal equivalente. Porém, como o número de dimensões é diretamente proporcional à incerteza contida no problema, os mercados não poderiam ser considerados completos, o que implica a existência de diferentes medidas martingais equivalentes. Para solucionar este problema, Gerber e Shiu (1994) se valem da transformada de Esscher. Em seu artigo, os autores, além de relaxarem a hipótese de mercados completos, consideram distribuições de probabilidade diferentes da normal, ou a binomial, para os retornos dos ativos, como, por exemplo, os processos de Poisson, Gamma e Gaussiano inverso. Já para o caso americano, Gerber e Shiu (1996) estendem os resultados de Margrabe utilizando o Teorema da Amostragem Opcional.

Mais recentemente, Fajardo e Mordecki (2003) apreçam derivativos cujos ativos subjacentes tenham seus preços modelados por processos de Lévy Geométricos, ou seja, eles generalizam o trabalho de Gerber e Shiu (1996) para o caso americano perpétuo. Cabe ressaltar que o caso americano finito ainda é um problema em aberto.

O objetivo deste trabalho é apresentar as metodologias usadas por Margrabe (1978) e Rubinstein (1991a) para o apreçamento de derivativos bidimensionais, aplicando-as a dados reais de produtos negociados no exterior e, especialmente, no Brasil, comparando as vantagens e desvantagens 
destas metodologias no apreçamento de tais instrumentos, e sugerir a adaptação destas metodologias a dois produtos de correlação de segunda ordem negociados na Bolsa de Valores de São Paulo, bem como a incorporação de produtos negociados no exterior ao leque de produtos derivativos oferecidos tanto pela Bolsa de Valores de São Paulo como pela Bolsa de Mercadorias \& Futuros.

$\mathrm{O}$ artigo está dividido da seguinte forma: na seção 2 faremos uma taxonomia das opções bidimensionais, na qual são apresentados exemplos de contratos negociados no exterior e no Brasil, tanto em mercados organizados de bolsa ou de balcão como mercados de balcão não-organizados. Na seção 3 será feita uma revisão das metodologias de apreçamento destes instrumentos. A seção 4 é dedicada à apresentação dos resultados obtidos usando dados reais. Por último são apresentadas as conclusões e um apêndice com as rotinas em Matlab® utilizadas na obtenção dos resultados.

\section{A TAXONOMIA E A UTILIZAÇÃo PRÁtiCA DOS DERIVATIVOS BIDIMENSIONAIS}

Nesta seção mostraremos uma classificação dos diferentes tipos de contratos derivativos bidimensionais existentes, listaremos aplicações práticas destas estruturas teóricas, isto é, arranjos financeiros que possuam seu pay-off dependente de dois ativos subjacentes e que sejam negociados tanto em pregão como em mercados de balcão, organizados ou não.

\subsection{Opções de troca ${ }^{1}$}

\subsubsection{Opção para trocar um ativo por outro}

$$
w\left(S_{1}, S_{2}, T\right)=\left(S_{2 T}-S_{1 T}\right)^{+}
$$

Uma aplicação direta deste tipo de derivativo seria utilizá-lo para monetizar as apostas de movimentos relativos de preços que são feitas no Brasil entre ações preferenciais nominativas e ordinárias nominativas. Estas opções são essencialmente, como veremos a seguir, opções de spread com offset igual a zero.

\subsubsection{Opção do melhor de dois ativos}

$$
w\left(S_{1}, S_{2}, T\right)=\max \left(S_{1 T}, S_{2 T}\right)
$$

\subsubsection{Opção do pior de dois ativos}

$$
w\left(S_{1}, S_{2}, T\right)=\min \left(S_{1 T}, S_{2 T}\right)
$$

Estes dois últimos tipos de opções servem a investidores que não possuem opinião definida sobre o movimento relativo de preços, e como podemos observar abaixo elas possuem suas funções de pagamento atreladas ao pay-off de uma opção de troca. Se não vejamos:

$$
\begin{aligned}
& \max \left(S_{I T}, S_{2 T}\right)=S_{I T}+\left(S_{2 T}-S_{I T}\right)^{+} \text {ou } S_{2 T}+\left(S_{I T}-S_{2 T}\right)^{+} \\
& \min \left(S_{I T}, S_{2 T}\right)=S_{1 T}-\left(S_{I T}-S_{2 T}\right)^{+} \text {ou } S_{2 T}-\left(S_{2 T}-S_{I T}\right)^{+}
\end{aligned}
$$

1 São sinônimos as seguintes expressões: margrabe option, exchange option e difference option. 
Pode-se perceber que a diferença entre as duas opções está na titularidade de uma opção de troca, e para cada uma existe um tipo de nota, negociada nos EUA, que serve para hedge ou alavancagem de posições em diferentes mercados. Nestes instrumentos o investidor obtém, como retorno um porcentual do máximo, ou do mínimo, entre dois ativos diferentes. Especificamente, estas notas são definidas conforme abaixo:

$$
\begin{aligned}
& B o T\left(\omega, V_{1}, V_{2}\right)=\omega \max \left(V_{1}, V_{2}\right) \\
& \operatorname{DIB}\left(\psi, V_{1}, V_{2}\right)=\psi \min \left(V_{1}, V_{2}\right)
\end{aligned}
$$

Embutida na DIB note está a venda de uma opção de troca. O prêmio da opção é utilizado para aumentar a alavancagem da nota, enquanto que embutida na BoT note está a compra de uma opção de troca. O prêmio da opção, neste caso, é utilizado para diminuir a alavancagem da nota.

\subsubsection{Opção composta}

\subsubsection{Razão call e put}

$$
\begin{aligned}
& c\left(S_{1}, S_{2}, T, K\right)=\left(S_{2 T} / S_{1 T}-K\right)^{+} \\
& p\left(S_{1}, S_{2}, T, K\right)=\left(K-S_{2 T} / S_{1 T}\right)^{+}
\end{aligned}
$$

\subsubsection{Produto call e put}

$$
\begin{aligned}
& c\left(S_{1}, S_{2}, T, K\right)=\left(S_{1 T} S_{2 T}-K\right)^{+} \\
& p\left(S_{1}, S_{2}, T, K\right)=\left(K-S_{1 T} S_{2 T}\right)^{+}
\end{aligned}
$$

\subsection{Opções com risco cambial ou FX-linked options}

As fx-linked options ou currency-translated options, como também são conhecidas, servem como instrumentos de proteção, em diferentes níveis, contra movimentos adversos em taxas de câmbio assim como preço de ativos. Esta classe de opções envolve invariavelmente um componente de taxa de câmbio cuja função é agir como numerário, isto é, um fator de conversão usado para mudar as unidades monetárias de uma moeda para outra. As fx-linked options são divididas em:

- Cross-currency options;

- Quantity-adjusted options (Quanto);

- Composite options (Compo);

\subsubsection{Cross currency}

Exemplos de cross-currency options são as opções sobre títulos nos quais o prêmio, o preço de exercício e o pay-off são denominados numa moeda diferente da moeda na qual os cupons dos títulos e o principal são pagos. Logo, o pay-off de uma cross-currency é dependente de dois fatores de risco: dos preços dos títulos e das taxas de câmbio. O primeiro uso deste tipo de derivativo foi feito 
por companhias de seguros, no Japão, que possuíam US Treasury Notes e Bonds em carteiras baseadas em ienes. Estes investidores queriam tanto comprar opções de venda para proteger o valor de suas carteiras como vender opções de compra em programas buy-write.

\subsubsection{Quanto}

Aplicações de quanto options encontram-se na aquisição de um ativo numa moeda diferente da moeda doméstica do comprador. Claramente, podemos inferir duas variantes para este tipo de derivativo, uma com taxa de câmbio fixa e outra com taxa de câmbio flutuante.

\subsubsection{Quanto com taxa de câmbio fixa}

Aqui, o comprador de uma opção numa ação estrangeira quer que a opção seja exercida na moeda estrangeira, mas prefere que o pay-off final seja convertido para sua moeda doméstica a uma taxa de câmbio preestabelecida. Assim, o investidor se protege de movimentos adversos na taxa de câmbio, mas simultaneamente deixa de ser favorecido por movimentos de apreciação da moeda doméstica em relação à moeda estrangeira. ${ }^{2}$ Então, o pay-off de uma quanto com taxa de câmbio fixa, isto é, previamente estabelecida sobre a ação denominada em moeda estrangeira e exercida na mesma moeda, com o pagamento sendo feito na moeda doméstica, é:

$$
\begin{aligned}
& c\left(S_{E}^{0}, S_{F}, K_{F}\right)=S_{E}^{0}\left(S_{F}-K_{F}\right)^{+} \\
& p\left(S_{E}^{0}, S_{F}, K_{F}\right)=S_{E}^{0}\left(K_{F}-S_{F}\right)^{+}
\end{aligned}
$$

O valor desta opção depende, obviamente, da correlação entre o preço da ação estrangeira e da taxa de câmbio. Como as taxas de câmbio são, na prática, flutuantes, o apreçamento de quanto préfixada requer um ajuste ao preço a termo da ação estrangeira por um fator proporcional ao diferencial de taxa de juros acumulado ao longo da vida da opção. Adicionalmente, como a taxa de câmbio e a ação são correlacionadas, um fator extra precisa ser introduzido no preço a termo.

\subsubsection{Quanto com taxa de câmbio flutuante}

Aqui, a taxa de câmbio de conversão não é fixa e sim a taxa prevalecente no dia de exercício. Esta quanto flexível, obviamente, não oferece proteção contra o risco cambial. No pay-off, final, o resultado é convertido por $S_{E}{ }^{T}$. O apreçamento utiliza o modelo de Black e Scholes com $S_{F}$ e $K_{F}$ e multiplicada por $S_{E}^{T}$, ou seja:

$$
\begin{aligned}
& c\left(S_{E}^{T}, S_{F}, K_{F}\right)=S_{E}^{T}\left(S_{F}-K_{F}\right)^{+} \\
& p\left(S_{E}^{T}, S_{F}, K_{F}\right)=S_{E}^{T}\left(K_{F}-S_{F}\right)^{+}
\end{aligned}
$$

\subsubsection{Compo}

São instrumentos, em contraste com as quanto options, sobre ações estrangeiras (denominados ou em moeda local ou em moeda estrangeira) exercidos tanto em moeda local como em moeda estrangeira. Existem duas variantes:

2 Para este fim existem os chamados BEACH (Best Equity-Adjusted Currency Hedge) options. 


\subsubsection{Opções em ações estrangeiras exercidas em moeda doméstica}

$$
\begin{aligned}
& c\left(S_{E}^{T}, S_{F}, K\right)=\left(S_{E}^{T} S_{F}-K\right)^{+} \\
& p\left(S_{E}^{T}, S_{F}, K\right)=\left(K-S_{E}^{T} S_{F}\right)^{+}
\end{aligned}
$$

Como o ativo subjacente neste instrumento é o produto $S_{E}{ }^{T} S_{F}$ a opção dependerá da correlação entre a taxa de câmbio e a ação estrangeira. Portanto, esta estrutura provê proteção contra o risco na taxa de câmbio, já que o efeito de correlação é explicitamente levado em consideração durante a avaliação da opção. Tratando-se o produto $S_{E}{ }^{T} S_{F}$ como um ativo, ela é simplesmente a opção de Margrabe para trocar um montante de moeda local $K$ com ações do ativo estrangeiro denominadas na moeda doméstica $S_{E}{ }^{T} S_{F}$

\subsubsection{Equity-linked foreign-exchange option}

$$
\begin{aligned}
& c\left(S_{E}^{T}, S_{F}, K\right)=S_{F}\left(S_{E}^{T}-K\right)^{+} \\
& p\left(S_{E}^{T}, S_{F}, K\right)=S_{F}\left(K-S_{E}^{T}\right)^{+}
\end{aligned}
$$

Esta opção é, na verdade, uma opção de taxa de câmbio que coloca um piso na exposição cambial do investidor. O investidor está exposto à alta e queda da ação estrangeira. Então, o investidor está exposto ao downside risk da ação, embora completamente protegido contra o downside risk da taxa de câmbio devido ao piso. Em outras palavras, é um investimento que combina uma opção de câmbio com um termo de ação. O titular tem o direito de comprar ou vender uma ação estrangeira com moeda doméstica, que pode ser convertida da moeda estrangeira usando um preço de taxa de câmbio de exercício, expresso em unidades de moeda doméstica por unidades de moeda estrangeira.

\section{Observação}

No Brasil, existe uma variante que combina uma opção sobre uma ação com a taxa de câmbio. Nessa modalidade, o preço de exercício é expresso em pontos, sendo que cada ponto equivale a um centésimo da taxa de câmbio real por dólar norte-americano, divulgada pelo Banco Central do Brasil. Todas as negociações nesse segmento, tanto com relação aos prêmios pagos e recebidos quanto aos montantes de exercícios, são efetuadas em reais. Outra particularidade desse tipo de opção é a existência de séries protegidas e não protegidas. As séries protegidas, assim como acontece com as opções vanilla, têm seus preços de exercício ajustados sempre que a empresa emissora das açõesobjeto distribui algum provento. Já as séries não protegidas somente são ajustadas no caso de proventos envolvendo ações do mesmo tipo (bonificações, desdobramentos e grupamentos).

Além desta, existe uma modalidade denominada Opções em IGP-M, que possui a particularidade de ter seu preço de exercício corrigido diariamente, a partir do dia de abertura da série, inclusive, até o dia de exercício, exclusive. A correção é feita pela multiplicação do valor em moeda local do dia por um fator de correção que corresponde à taxa de variação do IGP-M do mês anterior "pró-rateada" pelos dias úteis do mês corrente. ${ }^{3} \mathrm{O}$ apreçamento destes dois tipos de derivativos é feito utilizando-se o modelo de original de Black-Scholes com uma adaptação diferente para cada caso. Como a questão básica do apreçamento de opções de troca é como avaliar opções para as

3 As especificações contratuais deste tipo de opção podem ser encontradas em www.bovespa.com.br. 
quais o preço de exercício é incerto, e sendo este o caso para as opções com preços de exercício corrigido pela variação cambial bem como por algum índice de preços, um dos objetivos do texto será, exatamente, sugerir uma nova metodologia de apreçamento de tais instrumentos.

\subsection{Opções arco-íris}

\subsubsection{Opção multi-index}

$$
\begin{aligned}
& \max \left(S_{1 T}, S_{2 T}, X\right)=X+\left(\max \left(S_{1 T}, S_{2 T}\right)-X\right)^{+} \\
& \min \left(S_{1 T}, S_{2 T}, X\right)=X-\left(X-\min \left(S_{1 T}, S_{2 T}\right)\right)^{+}
\end{aligned}
$$

\subsubsection{Opções sobre o máximo ou mínimo}

$$
\begin{array}{lll}
\left(\max \left(S_{1 T}, S_{2 T}\right)-K\right)^{+} & \text {ou } & \max \left(S_{1 T}, S_{2 T}, K\right)-K \\
\left(K-\max \left(S_{1 T}, S_{2 T}\right)\right)^{+} & \text {ou } & \left(\max \left(S_{1 T}, S_{2 T}\right)-K\right)^{+}-\max \left(S_{1 T}, S_{2 T}\right)+K \\
\left(\min \left(S_{1 T}, S_{2 T}\right)-K\right)^{+} & \text {ou } & \left(S_{1 T}-K\right)^{+}+\left(S_{2 T}-K\right)^{+}-\left(\max \left(S_{1 T}, S_{2 T}\right)-K\right)^{+} \\
\left(K-\min \left(S_{1 T}, S_{2 T}\right)\right)^{+} & \text {ou } & \left(\min \left(S_{1 T}, S_{2 T}\right)-K\right)^{+}-\min \left(S_{1 T}, S_{2 T}\right)+K
\end{array}
$$

Foi Stulz (1982) quem derivou as soluções analíticas para o problema das opções de máximo e de mínimo sobre dois ativos de risco. Posteriormente, suas soluções foram generalizadas para o caso de $n$ ativos de risco. ${ }^{4}$ A aplicação direta deste tipo de opção consiste no hedge de carteiras com ativos com correlações negativas, sendo a Bond-Over-Stock (BOS) Warrant do Bankers Trust International um exemplo prático deste derivativo.

Cabe ainda mencionar que, em conjunto, as opções de máximo e de mínimo são referidas como min-max options, enquanto que as opções de melhor ou pior entre $n$ ativos, cujas respectivas traduções para a língua inglesa são best-of e worst-of, são referidas coletivamente como alternative options.

\subsubsection{Opção de spread}

$$
\begin{aligned}
& c\left(S_{1}, S_{2}, T, K\right)=\left(\left(S_{2 T}-S_{1 T}\right)-K\right)^{+} \\
& p\left(S_{1}, S_{2}, T, K\right)=\left(K-\left(S_{2 T}-S_{1 T}\right)\right)^{+}
\end{aligned}
$$

Pode-se perceber que se $K$ for igual a zero, estas opções se tornam opções de troca. Exemplos de aplicações práticas deste tipo de opção são encontrados na família de opções de spread desenvolvidas pela Goldman Sachs para investidores em mercados de renda fixa. Abaixo discorremos brevemente sobre cada uma destas estruturas:

4 Ver Johnson (1987) 
- Slope-of-the-Yield-Curve Options (SYCURVE) $:^{5}$ servem para a negociação do formato da estrutura a termo de taxas de juros na medida em que oferecem a oportunidade para comprar ou vender a yield curve. Comprar a yield curve significa comprar o vencimento curto e vender o vencimento longo com duração casada. Vender a yield curve é a estratégia reversa, ou seja, vender o vencimento curto e comprar o vencimento longo com duração casada. Exemplos são as opções do spread entre os US Treasuries de dois anos contra os US Treasuries de dez e de trinta anos, podendo ser do tipo europeu ou americano e sendo o preço de exercício cotado como um spread de yield ${ }^{6}$ em pontos-base.

- Mortgage Over Treasury Options (MOTTO): são desenhadas para investidores que querem negociar o risco de mudanças no spread entre hipotecas e os títulos do governo americano. $\mathrm{O}$ comprador de uma call motto se beneficia quando os Mortgage Backed Securities possuem um desempenho melhor que os Treasuries e o comprador de uma put motto quando acontece o contrário. Devido à inexistência, no mercado de ativos hipotecários, de uma correspondência biunívoca de preço e yield, por serem os futuros fluxos de caixa de um ativo hipotecário desconhecido, ${ }^{7}$ a liquidação é sempre física, ou seja, no caso de uma call motto, seu detentor compra determinada quantidade de MBS e vende uma quantidade predeterminada de Treasuries a um preço de exercício também predeterminado; já no caso de uma put motto, o detentor vende determinada quantidade de MBS e compra uma quantidade predeterminada de Treasuries a um preço de exercício também predeterminado.

- International Spread Options (ISO): são iguais as sycurve, mas os ativos subjacentes são denominados em moedas diferentes. A moeda na qual o comprador da opção é pago quando a opção expira dentro do dinheiro é previamente especificada e esta é a moeda na qual a opção é denominada. Também tal como as sycurve, os pay-offs são cotados em pontos-base.

- Spread-Lock Options: são opções sobre swaps de taxas de juros. O detentor da call tem o direito de comprar um swap de taxas de juros (receber um montante fixo e pagar um montante flutuante) a um spread predeterminado, enquanto que detentor da put tem o direito de vender um swap de taxas de juros (pagar um montante fixo e receber um montante flutuante) a um spread predeterminado. Diferenciam-se de swaptions, caps e floors já que estes são opções sobre níveis de taxas de juros e não sobre spreads de taxas de juros.

\section{Observação}

No Brasil, a aplicação das spread-lock options é direta, em especial no caso dos swaps negociados na Bolsa de Mercadorias \& Futuros, na qual são admitidos à negociação dezenove tipos de variáveis, conforme abaixo:

- PRE: Taxa pré-fixada.

- DI1: Taxa Média de Depósitos Interfinanceiros de Um Dia (DI), divulgada pela CETIP.

- DOL: Taxa de câmbio de R $\$$ por US\$ dos EUA, no segmento de taxas livremente pactuadas, divulgada pelo BCB.

- TR: Taxa Referencial, divulgada pelo BCB.

- IGP: Índice de preços.

5 "Sycurve Options: Puts and Calls on the Slope of the Yield Curve" (Goldman, Sachs \& Co., Novembro 1989).

6 Como os pay-offs são determinados por spreads de yields e não pelos níveis de yields as durations e convexidades dos dois ativos são, aproximadamente, zero. Isto porque um deslocamento paralelo na estrutura a termo de taxas de juros, isto é, a yield curve esperada para a data de exercício da opção não afetará os valores das opções, mas um deslocamento paralelo na yield curve corrente não necessariamente implica um deslocamento paralelo na yield curve a termo e, portanto, não se pode garantir que mudanças na yield curve corrente não implicam mudanças na exposição aos níveis de yield nas opções.

7 Isto se deve ao fato de não se conhecer as taxas de pré-pagamento das hipotecas individuais que lastreiam o ativo. 
- OZ1: Preço do ouro, negociado no mercado disponível da BM\&F.

- SEL: Taxa Média Ajustada dos Financiamentos Diários Apurados no SELIC para os Títulos Públicos Federais.

- TBF: Taxa Básica Financeira, divulgada pelo BCB.

- ANB: Taxa média de depósitos a prazo, divulgada pela ANBID.

- IND: Índice de ações da BOVESPA (IBOVESPA).

- TJL: Taxa de Juro de Longo Prazo (TJLP), divulgada pelo CMN.

- SB1: Carteira de ações (Stock Basket 1), constituída de ações negociadas na BOVESPA, dentre as autorizadas pela $\mathrm{BM} \& \mathrm{~F}$.

- SB2: Carteira de ações (Stock Basket 2), constituída de ações negociadas na BOVESPA, dentre as autorizadas pela $\mathrm{BM} \& \mathrm{~F}$.

- REU: Taxa de câmbio de reais por euro.

- IGM: Índice Geral de Preços-Mercado (IGP-M), calculado pelo IBRE da FGV.

- IGD: Índice Geral de Preços-Disponibilidade Interna (IGP-M), calculado pelo IBRE da FGV.

- IPC: Índice de Preços ao Consumidor (IPC), calculado pela FIPE da USP.

- INP: Índice Nacional de Preços ao Consumidor (INPC), calculado pelo IBGE.

- IAP: Índice Nacional de Preços ao Consumidor Amplo (IPCA), calculado pelo IBGE.

Tabela 1

\begin{tabular}{|c|c|c|c|c|c|c|c|c|c|c|c|c|c|c|c|c|c|c|c|c|}
\hline & 1.PRE & 2.DI1 & 3.DOL & 4.TR & $5 . \mathrm{IGP}$ & $6.0 Z 1$ & 7.SEL & 8.TBF & 9.ANB & 10.IND & 11.TJL & 12.SB1 & 12.SB2 & 14.REU & 15.IGM & 16.IGD & 17.IPC & 18.INP & 19.IAP & 20.JPY \\
\hline 1.PRE & \begin{tabular}{|l} 
\\
\end{tabular} & - & - & - & - & - & - & - & - & - & - & - & - & - & - & - & - & - & - & - \\
\hline 2.DI1 & SDP & - & - & - & - & - & - & - & - & - & - & - & - & - & - & - & - & - & - & - \\
\hline 3.DOL & SCP & SDC & - & - & - & - & - & - & - & - & - & - & - & - & - & - & - & - & - & - \\
\hline 4.TR & STP & SDT & SCT & - & - & - & - & - & - & - & - & - & - & - & - & - & - & - & - & - \\
\hline $5 . I G P$ & SGP & SDG & SGC & $\overline{\text { STG }}$ & - & - & - & - & - & - & - & - & - & - & - & - & - & - & - & - \\
\hline $6.0 Z 1$ & SOP & SDO & $\mathrm{SCO}$ & STO & SGO & - & - & - & - & - & - & - & - & - & - & - & - & - & - & - \\
\hline 7.SEL & SSP & SDS & SSC & SST & SSG & SSO & - & - & - & - & - & - & - & - & - & - & - & - & - & - \\
\hline 8.TBF & SBP & SDB & SBC & STB & SBG & SBO & SBS & - & - & - & - & - & - & - & - & - & - & - & - & - \\
\hline 9.ANB & SAP & SDA & SCA & STA & SAG & SAO & SAS & SBA & - & - & - & - & - & - & - & - & - & - & - & - \\
\hline 10.IND & SNP & SDN & SCN & STN & SNG & SNO & SNS & SBN & SNA & - & - & - & - & - & - & - & - & - & - & - \\
\hline 11.TJL & SJP & SDJ & SCJ & STJ & SJG & SJO & SJS & SBJ & SJA & SNJ & - & - & - & - & - & - & - & - & - & - \\
\hline 12.SB1 & SPB & SBD & SCB & SBT & SGB & $\mathrm{SOB}$ & SSB & SBB & SAB & SNB & SJB & - & SBK & - & - & - & - & - & - & - \\
\hline $13 . \mathrm{SB} 2$ & - & - & - & - & - & - & - & - & - & - & - & SBK & - & - & - & - & - & - & - & - \\
\hline 14.REU & SEP & SDE & SCE & STE & - & SOE & SSE & SBE & SAE & SNE & SJE & SEB & - & - & - & - & - & - & - & - \\
\hline 15.IGM & SMP & SDM & SCM & STM & - & SOM & SSM & SBM & SAM & SNM & SJM & SMB & - & SME & - & - & - & - & - & - \\
\hline 16.IGD & SIP & SDI & $\mathrm{SCl}$ & STI & - & $\mathrm{SIO}$ & SSI & SBI & SAI & SNI & SJI & SIB & - & SEI & SMI & - & - & - & - & - \\
\hline 17.IPC & SPF & SFD & SFC & SFT & - & SFO & SFS & SFF & SFA & SFN & SJF & SDD & - & SEF & SMF & SIF & - & - & - & - \\
\hline 18.INP & SXP & SDX & SCX & STX & - & SOX & SSX & SBX & SAX & SNX & SJX & SXB & - & SXE & SMX & SIX & SFX & - & - & - \\
\hline 19.IAP & SLP & SDL & $\mathrm{SCL}$ & STL & - & $\mathrm{SOL}$ & SSL & SBL & SAL & SNL & SJL & SLB & - & SLE & SML & SIL & SFL & $S X L$ & - & - \\
\hline 20.JPY & SYP & SDY & SCY & STY & - & SOY & SSY & SBY & SAY & SNY & SJY & SYB & - & SYE & SMY & SIY & SFY & SXY & SLY & - \\
\hline
\end{tabular}

Pode-se observar que dentre as 19 variáveis admitidas sete são taxas de juros, seis são índices de inflação, duas são taxas de câmbio, três são variáveis ligadas ao mercado de renda variável e uma é o preço de uma commodity, o ouro. Da Tabela 1 podemos ver um potencial de possíveis combinações destas variáveis: são 165 tipos diferentes de spread-lock options considerando-se somente estes ativos.

A seguir apresentaremos as metodologias para o apreçamento de vários destes contratos. 


\section{MODELOS DE APREÇAMENTO}

Dentre os derivativos apresentados, determinados tipos possuem fórmulas analíticas fechadas de apreçamento enquanto outros não, isto é, seu apreçamento depende do emprego de métodos numéricos. Aqui, apresentamos a solução analítica de Margrabe para o problema das opções de troca bem como a solução numérica de Rubinstein para o mesmo problema, com a vantagem de poder ser aplicado, no Brasil, ao caso das opções vanilla com preços de exercício corrigidos por variação cambial.

\subsection{Modelo de Margrabe}

Formalmente, o problema da opção de troca é o seguinte:

$$
w=\left(S_{1}-S_{2}\right)^{+}
$$

Pode-se observar que uma opção de troca do ativo 2 pelo ativo 1, conforme (1), pode ser encarada tanto como uma call sobre o ativo 1 com preço de exercício flutuante, sendo o valor do ativo 2, quanto como uma put sobre o ativo 2 com preço de exercício flutuante, sendo o valor do ativo 1 . Também de (1) vemos que uma opção de troca nada mais é do que uma generalização do problema de uma opção vanilla, resolvido por Black e Scholes (1973) e Merton (1973), na qual o preço de exercício determinístico desta última é substituído por um processo estocástico e, portanto, as hipóteses usadas são as mesmas:

- A taxa de juros de curto prazo é conhecida e constante através do tempo;

- Não existem dividendos, juros sobre capital próprio ${ }^{8}$ ou bonificações; logo, todos os retornos advêm de ganhos de capital;

- O ativo é contingente, isto é, é uma opção européia, a qual somente pode ser exercida em sua data de vencimento;

- Os mercados são perfeitos, isto é, não existem friç̧ões, como custos de transação e tributação, tanto para os ativos subjacentes como para o derivativo;

- Além de perfeitos, os mercados são eficientes, isto é, os preços dos ativos subjacentes refletem plenamente a informação disponível;

- Os ativos subjacentes são perfeitamente divisíveis;

- É permitida a venda a descoberto.

\section{O Processo de Preço e a Condição Terminal}

Sejam dois ativos $(\mathrm{i}=1,2)$ e sejam $S_{1}$ e $S_{2}$ os preços dos ativos 1 e 2 , respectivamente. Como os processos de preços dos ativos subjacentes são erráticos, para modelar sua dinâmica assume-se que as taxas de retorno em cada ativo são dadas por um Movimento Browniano Geométrico: ${ }^{9}$

$$
\frac{d S_{i t}}{S_{i t}}=\mu_{i} d t+\sigma_{i} d z_{i t}
$$

\footnotetext{
8 Podem ser tratados como dividendos com a vantagem fiscal de serem contabilizados como despesa no balanço patrimonial.

9 Denotado simplesmente por MBG daqui em diante.
} 
Como pode ser visto, a taxa de retorno é um processo de Ito, e define-se a correlação entre os processos de Wiener $d z_{1}$ e $d z_{2}$ como $\rho_{12}$. O objetivo aqui é encontrar o valor de uma opção européia com vencimento no tempo em $T$ e cujo pay-off seja:

$$
\begin{aligned}
& c\left(S_{1}, S_{2}, T\right)=\left(S_{1 T}-S_{2 T}\right)^{+} \\
& p\left(S_{1}, S_{2}, T\right)=\left(S_{2 T}-S_{1 T}\right)^{+}
\end{aligned}
$$

A abordagem utilizada por Margrabe é a mesma de Black e Scholes, isto é, o da carteira equivalente, que será detalhada adiante. Antes, porém, de entrar na derivação propriamente dita, e já de posse da condição terminal da opção, é necessário estabelecer as condições limítrofes de preço, pois para se chegar à solução encontrada por Margrabe é preciso resolver uma equação diferencial parcial.

\section{Condição terminal do preço}

No tempo $t$, consideremos duas carteiras, conforme as Tabelas 2 e 3 abaixo:

\begin{tabular}{|c|c|c|c|c|}
\hline & & \multirow[b]{2}{*}{ Valor Presente } & \multicolumn{2}{|c|}{ Valor em T } \\
\hline & & & $\mathrm{S}_{1 \mathrm{~T}} \leq \mathrm{S}_{2 \mathrm{~T}}$ & $S_{1 T}>S_{2 T}$ \\
\hline $1^{\mathrm{a}}$ carteira & Opção de Troca do ativo 2 pelo ativo 1 & $\mathrm{c}_{\mathrm{t}}\left(\mathrm{S}_{1}, \mathrm{~S}_{2}\right)$ & 0 & $+S_{1 T} S_{2 T}$ \\
\hline \multirow[t]{2}{*}{$2^{\mathrm{a}}$ carteira } & $+S_{1}$ & $+S_{1 \mathrm{t}}$ & $+S_{1 T}$ & $+\mathrm{S}_{1 \mathrm{~T}}$ \\
\hline & $-S_{2}$ & $-S_{2 t}$ & $-S_{2 T}$ & $-S_{2 T}$ \\
\hline Total & & $+S_{1 t}-S_{2 t}$ & $+S_{1 T}-S_{2 T}$ & $+S_{1 T} S_{2 T}$ \\
\hline
\end{tabular}

Tabela 2

Tabela 3

\begin{tabular}{lcccc}
\hline & & & \multicolumn{2}{c}{ Valor em T } \\
\cline { 4 - 5 } & & Valor Presente & $S_{1 T} \leq S_{2 T}$ & $S_{1 T}>S_{2 T}$ \\
\hline $3^{\text {a }}$ carteira & Opção de Troca do ativo 1 pelo ativo 2 & $p_{t}\left(S_{2}, S_{1}\right)$ & $+S_{2 T}-S_{1 T}$ & 0 \\
$4^{\text {a carteira }}$ & $-S_{1}$ & $-S_{1 t}$ & $-S_{1 T}$ & $-S_{1 T}$ \\
& $+S_{2}$ & $+S_{2 t}$ & $+S_{2 T}$ & $+S_{2 T}$ \\
Total & & $+S_{2 T}-S_{1 T}$ & $+S_{2 T}-S_{1 T}$ & $+S_{2 T}-S_{1 T}$ \\
\hline
\end{tabular}

Como se pode notar, as carteiras com as opções são dominantes sobre as outras duas carteiras com as posições nos ativos e, portanto, o valor das opções não pode ser menor que o valor das carteiras com os ativos. Logo, ${ }^{10}$

10 Note que se a opção de troca fosse americana o exercício antecipado geraria somente $+\mathrm{S}_{1 \mathrm{t}}-\mathrm{S}_{2 \mathrm{t}}$ ou $+\mathrm{S}_{2 \mathrm{t}}-\mathrm{S}_{1 \mathrm{t}}$ e, portanto, não haveria prêmio pelo exercício antecipado. Logo, a opção americana deveria valer o mesmo que a européia. 


$$
\begin{aligned}
& c\left(S_{1}, S_{2}, t\right) \geq\left(S_{1 t}-S_{2 t}\right)^{+} \\
& p\left(S_{1}, S_{2}, t\right) \geq\left(S_{2 t}-S_{1 t}\right)^{+}
\end{aligned}
$$

\section{O apreçamento}

Como dissemos, para chegar a uma solução analítica Margrabe utilizou um conceito de carteira equivalente. Esta metodologia se baseia na idéia de preço racional, isto é, ela identifica qual seria o preço do derivativo sendo ele um ativo atingível, isto é, existiria uma carteira/estratégia autofinanciável ${ }^{11}$ que replicaria o valor de seu pay-off. Fica claro que Margrabe supunha mercados completos, isto é, seria possível obter o preço racional do derivativo considerando a hipótese de não-arbitragem combinada com a redundância do derivativo, que é conseqüência da própria hipótese de mercados completos.

Seja, então, $w=w\left(S_{1}, S_{2}\right)$. Assim, para se proteger uma posição assumida em uma opção de troca assume-se uma posição em $w_{1}$ unidades do ativo 1 e $w_{2}$ unidades do ativo $2{ }^{12}$ A fórmula de apreçamento de $w\left(\right.$.) deve ser linear em $S_{1}$ e $S_{2}$. Assim, utilizando-se o Teorema de Euler pode-se expressar o valor da opção como:

$$
w-w_{1} S_{1 t}-w_{2} S_{2 t}=0
$$

onde $w_{i}=\partial w / \partial S_{i},(i=1,2)$.

Logo, para que não seja possível arbitragem, tal carteira deve retornar a zero,

$$
d w-w_{1} d S_{1 t}-w_{2} d S_{2 t}=0
$$

Aplicando-se a versão multidimensional do Lema de Itô, o valor da opção é:

$$
d w=w_{1} d S_{1 t}+w_{2} d S_{2 t}+w_{3} d t+\frac{1}{2}\left(\frac{\partial^{2} w}{\partial S_{1 t}{ }^{2}} \sigma_{1}^{2} S_{1 t}^{2}+\frac{\partial^{2} 2 w}{\partial S_{1 t} S_{2 t}} \sigma_{1} \sigma_{2} \rho_{12} S_{1 t} S_{2 t}+\frac{\partial^{2} w}{\partial S_{2 t}{ }^{2}} \sigma_{2}{ }^{2} S_{2 t}^{2}\right)
$$

Substituindo (7) em (6), obtém-se a seguinte equação diferencial parcial,

$$
w_{3} d t+\frac{1}{2}\left(\frac{\partial^{2} w}{\partial S_{1 t}{ }^{2}} \sigma_{1}^{2} S_{1 t}^{2}+\frac{\partial^{2} 2 w}{\partial S_{1 t} S_{2 t}} \sigma_{1} \sigma_{2} \rho_{12} S_{1 t} S_{2 t}+\frac{\partial^{2} w}{\partial S_{2 t}{ }^{2}} \sigma_{2}{ }^{2} S_{2 t}^{2}\right)=0
$$

Sua solução é:

$$
w\left(S_{1 t} S_{2 t}\right)=S_{1 t} N\left(d_{1}\right)-S_{2 t} N\left(d_{2}\right)
$$

onde,

11 São carteiras/estratégias que não recebem recursos adicionais ao longo do tempo e reinvestem todos os ganhos obtidos.

12 Como esta é a carteira autofinanciável, não deve requerer qualquer investimento inicial. 


$$
d_{1}=\frac{\ln \left(S_{1 t} / S_{2 t}\right)+\frac{1}{2} \sigma^{2}(T-t)}{\sigma \sqrt{T-t}} \quad d_{2}=d_{1}-\sigma \sqrt{T-t}
$$

e

$$
\sigma^{2}=\sigma_{1}^{2}-2 \sigma_{1} \sigma_{2} \rho_{12}+\sigma_{2}^{2}
$$

é a volatilidade de $\ln \left(S_{1} / S_{2}\right)$.

A solução da equação (9) satisfaz (1), (3), (8) e é única.

\section{Opções americanas}

A equação (9) também fornece o preço para as opções americanas se $S_{1}$ e $S_{2}$ são preços de equilíbrio dos ativos 1 e 2. Para provar isto, basta considerar, como no estabelecimento dos limites inferiores de preço, duas carteiras conforme tabela abaixo,

\section{Tabela 4}

\begin{tabular}{cccc}
\hline & & Valor Presente & Valor em T \\
\hline $1^{\text {a }}$ carteira & Opção de Troca do ativo 2 pelo ativo 1 & $\mathrm{w}\left(\mathrm{S}_{1 \mathrm{t}} \mathrm{S}_{2 \mathrm{t}}\right)$ & $\left(\mathrm{S}_{1 \mathrm{~T}}-\mathrm{S}_{2 \mathrm{~T}}\right)^{+}$ \\
$\mathbf{2}^{\mathrm{a}}$ carteira & $+\mathrm{S}_{1}-\mathrm{S}_{2}$ & $\mathrm{~S}_{1 \mathrm{t}}-\mathrm{S}_{2 \mathrm{t}}$ & $\mathrm{S}_{1 \mathrm{~T}}-\mathrm{S}_{2 \mathrm{~T}}$ \\
\hline
\end{tabular}

Como a carteira 1 é dominante sobre a carteira 2, então 1 deve valer pelo menos tanto quanto 2:

$$
w\left(S_{1 t} S_{2 t}\right) \geq S_{1 t}-S_{2 t}
$$

Logo, o valor de uma opção européia excede o que se conseguiria se exercêssemos uma opção americana. Então,

$$
W\left(S_{1 t}, S_{2 t}\right)=w\left(S_{1 t}, S_{2 t}\right)
$$

\begin{tabular}{|c|c|c|c|c|}
\hline & \multirow[b]{2}{*}{ Instrumento } & \multirow[b]{2}{*}{ Valor Presente } & \multicolumn{2}{|c|}{ Valor em T } \\
\hline & & & $\mathrm{S}_{1 \top} \leq \mathrm{S}_{2 T}$ & $S_{1 T}>S_{2 T}$ \\
\hline \multirow[t]{2}{*}{$1^{\mathrm{a}}$ carteira } & Opção de Troca do ativo 2 pelo ativo 1 & $c_{t}\left(S_{1}, S_{2}\right)$ & 0 & $+S_{1 T}-S_{2 T}$ \\
\hline & Opção de Troca do ativo 1 pelo ativo 2 & $-p_{t}\left(S_{2}, S_{1}\right)$ & $+S_{2 T}-S_{1 T}$ & 0 \\
\hline Total & & $c_{t}\left(S_{1}, S_{2}\right)-p_{t}\left(S_{2}, S_{1}\right)$ & $+S_{1 T}-S_{2 T}$ & $+S_{1 \top}-S_{2 T}$ \\
\hline \multirow[t]{2}{*}{$2^{\mathrm{a}}$ carteira } & Posição comprada no ativo 1 & $+S_{1 \mathrm{t}}$ & $+S_{1 T}$ & $+S_{1 \top}$ \\
\hline & Posição vendida no ativo 2 & $-S_{2 t}$ & $-S_{2 T}$ & $-S_{2 T}$ \\
\hline Total & & $+S_{1 t}-S_{2 t}$ & $+S_{1 T}-S_{2 T}$ & $+S_{1 T}-S_{2 T}$ \\
\hline
\end{tabular}

Paridade entre calls e puts

De forma análoga, pode-se obter uma relação de paridade entre calls e puts:

\section{Tabela 5}


Aqui as carteiras 1 e 2 produzem os mesmos pay-offs; logo, seus valores iniciais devem ser os mesmos. Conseqüentemente,

$$
c\left(S_{1,}, S_{2 t}\right)-p\left(S_{2 t}, S_{1 t}\right)=+S_{1 t}-S_{2 t}
$$

\subsection{Modelos lattice para avaliação de derivativos bidimensionais}

Desde que Cox, Ross e Rubinstein (CRR) publicaram, em 1979, seu trabalho sobre o apreçamento de opções utilizando um método lattice, extensões e generalizações de tal modelo foram desenvolvidas, em especial aquelas destinadas à avaliação de derivativos multidimensionais. Dentre estas extensões estão os trabalhos de Boyle (1988), Boyle, Evnine e Gibbs (1989), Kamrad e Ritchken (1991) e Rubinstein (1991) e é exatamente de tais artigos que estaremos tratando nesta parte do texto.

O modelo CRR assumia que o preço do ativo subjacente seguisse um processo binomial multiplicativo ao longo de sucessivos intervalos de tempo discretos. Além disto, o fato de as probabilidades de ocorrência de movimentos ascendentes e descendentes de preços não constarem das fórmulas de apreçamento implica que mesmo se investidores diferentes possuírem diferentes probabilidades subjetivas sobre tais movimentos, ainda assim poderiam concordar sobre a relação entre o prêmio da opção, o preço do ativo-objeto e suas taxas de retorno e a taxa de juros livre de risco. Assim, o valor do derivativo não dependeria das atitudes dos investidores em relação ao risco. Em resumo, o principal resultado do modelo é mostrar que o valor de uma opção pode ser interpretado como a expectativa de seu valor futuro descontado num mundo neutro ao risco.

Boyle (1988) foi o primeiro a utilizar a abordagem de CRR para a avaliação de contratos bidimensionais. A idéia básica utilizada foi a mesma, ou seja, se conhecermos as hipóteses sobre a distribuição de probabilidade dos preços dos ativos subjacentes, e se nos certificamos de que um apreçamento neutro ao risco é apropriado, então aproximações discretas podem ser utilizadas. A diferença consistiu na substituição do processo de duplo salto por um processo trinomial, o que, para o problema bidimensional, resultou em um processo com cinco resultados possíveis. Como premissa básica, Boyle assumia a distribuição de probabilidade dos preços dos dois ativos subjacentes como lognormal bivariada. Assim, com a utilização de uma avaliação neutra ao risco, ambos os ativos deveriam render a taxa de juros livre de risco e, portanto, para especificar a distribuição de probabilidades era necessária a matriz de variâncias e covariâncias. Logo, como a distribuição de probabilidade discreta deveria possuir os mesmos valores esperados e de variâncias e covariância da distribuição de probabilidade original, então seriam necessários, no mínimo, cinco graus de liberdade para a construção da distribuição de probabilidade discreta. Além disso, era necessário assegurar que as probabilidades dos saltos somassem um, o que seria um outro condicionante. Portanto, a construção do processo de cinco saltos mencionada no parágrafo anterior é justificada, e o único problema a ser enfrentado era como obter, nos moldes de CRR, as probabilidades (não negativas) e amplitudes dos saltos de preços para os dois ativos subjacentes.

Foi exatamente o problema de obtenção dos valores não negativos para as probabilidades neutras ao risco que o próprio Boyle, em conjunto com Evnine e Gibbs, atacaram um ano depois. Em seu trabalho, eles constroem uma distribuição de probabilidade discreta para aproximar a distribuição de probabilidade lognormal multivariada, e para isto escolhem os tamanhos e probabilidades dos saltos de tal forma que a função característica das duas distribuições de probabilidade convirja. Eles propuseram um modelo que, para problemas bidimensionais, utiliza uma lattice de quatro saltos. 
Kamrad e Ricthken (1991) atendem, da forma mais objetiva e generalizada possível, à demanda por métodos de avaliação de derivativos multidimensionais com a utilização de modelos lattice. Em seu artigo, os autores utilizam o fato de que prover uma ligação direta entre o processo de preços e a estratégia de arbitragem não é essencial; na verdade, se os mercados são completos e não existem oportunidades de arbitragem, então uma medida de probabilidade martingal equivalente existe, e esta medida permite a avaliação de qualquer ativo contingente por meio do cálculo de uma expectativa condicional apropriada. ${ }^{13}$ Além disso, seu principal resultado é um modelo multinomial, o qual permite, ao contrário do modelo de Boyle, Evnine e Gibbs, saltos horizontais de preços.

Simultaneamente, Rubinstein provém uma derivação binomial para o resultado encontrado por Margrabe. Como no caso de opções vanilla, a abordagem binomial clarifica a intuição econômica que está por detrás da fórmula de Margrabe podendo lidar com a possibilidade de exercício antecipado das opções americanas, e é com este intuito que apresentamos a solução obtida por Rubinstein.

\section{O modelo binomial de 2 periodos de Rubinstein}

A princípio, pode parecer que uma abordagem binomial não funcionaria, já que após o primeiro período de tempo existem quatro possíveis resultados:

- $S_{1}$ e $S_{2}$ sobem

- $S_{1}$ e $S_{2}$ descem

- $S_{1}$ sobe e $S_{2}$ desce

- $S_{1}$ desce e $S_{2}$ sobe

No limite de tal processo, à medida que $\delta t \rightarrow 0$, isto é, à proporção que os intervalos de tempo entre cada período tendem a zero, o que se quer é que a distribuição das duas variáveis seja univariada lognormal. Isto sugere o primeiro insight do autor, que é modelar a razão de preços entre os dois ativos como binomial univariada. Assim, é proposto um ajuste que resulta em dois resultados possíveis em vez de quatro.

Redefinindo a equação (1) para

$$
w=S_{1}\left[S_{2} / S_{1}-1\right]^{+}
$$

a árvore binomial para dois períodos seria

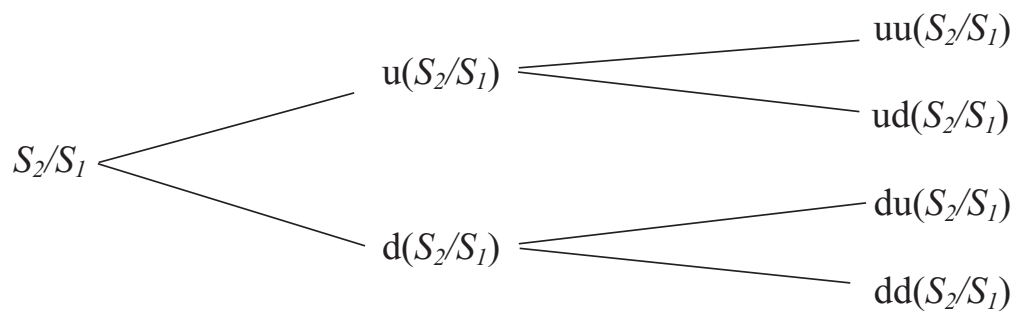

onde,

$u=1+$ taxa de crescimento dos preços relativos dos dois ativos;

$d=1+$ taxa de decrescimento dos preços relativos dos dois ativos.

13 Ver Cox e Ross (1976) e Harisson e Pliska (1981). 
É óbvio que os caminhos de preço relativo ud e du terminam no mesmo ponto e que u $>1$ e $\mathrm{d}<1$. Pode-se ver também que,

$$
\begin{aligned}
& w_{u u}=S_{u u 1}\left[u u\left(S_{2} / S_{1}\right)-1\right]^{+} \\
& w_{u d}=S_{u d 1}\left[u d\left(S_{2} / S_{1}\right)-1\right]^{+} \\
& w_{d u}=S_{d u 1}\left[d u\left(S_{2} / S_{1}\right)-1\right]^{+} \\
& w_{d d}=S_{d d 1}\left[d d\left(S_{2} / S_{1}\right)-1\right]^{+}
\end{aligned}
$$

Definindo-se

$$
\begin{aligned}
& R_{u u} \equiv\left[u u\left(S_{2} / S_{1}\right)-1\right]^{+} \\
& R_{u d} \equiv\left[u d\left(S_{2} / S_{1}\right)-1\right]^{+} \\
& R_{d u} \equiv\left[d u\left(S_{2} / S_{1}\right)-1\right]^{+} \\
& R_{d d}=\left[d d\left(S_{2} / S_{1}\right)-1\right]^{+}
\end{aligned}
$$

Então,

$$
\begin{aligned}
& w_{u u}=S_{u u l} R_{u u} \\
& w_{u d}=S_{u d l} R_{u d} \\
& w_{d u}=S_{d u l} R_{d u} \\
& w_{d d}=S_{d d 1} R_{d d}
\end{aligned}
$$

Cabe observar, antes de prosseguirmos, que estamos assumindo os dois ativos subjacentes como tendo uma taxa de dividendos constante a cada período, pois como o interesse é na avaliação de opções americanas, e como por (12) a troca de numerário iguala o problema da opção de troca ao de uma call americana vanilla, com o ativo subjacente tendo preço igual a $S_{2} / S_{1}$ e preço de exercício igual a 1 , não há ganho nesta abordagem caso os ativos não tenham dividendos.

Consideremos, agora, as duas situações possíveis durante o período 1:

movimento ascendente de $S_{2} / S_{1}$

$$
\begin{gathered}
W_{u u}=\Delta_{2} S_{u u 2} \delta_{2}+\Delta_{1} S_{u u 1} \delta_{1}=\Delta_{2} S_{u u 1} u u\left(S_{2} / S_{1}\right) \delta_{2}+\Delta_{1} S_{u u 1} \delta_{1} \\
W_{u d}=\Delta_{2} S_{u d 2} \delta_{2}+\Delta_{1} S_{u d 1} \delta_{1}=\Delta_{2} S_{u d l} u d\left(S_{2} / S_{1}\right) \delta_{2}+\Delta_{1} S_{u d l} \delta_{1} \\
W_{u u}=S_{u u 1}\left\{\Delta_{2} u u\left(S_{2} / S_{1}\right) \delta_{2}+\Delta_{1} \delta_{1}\right\}=S_{u u 1} R_{u u} \\
W_{u d}=S_{u d 1}\left\{\Delta_{2} u d\left(S_{2} / S_{1}\right) \delta_{2}+\Delta_{1} \delta_{1}\right\}=S_{u d l} R_{u d} \\
\Delta_{2} u u\left(S_{2} / S_{1}\right) \delta_{2}+\Delta_{1} \delta_{1}=R_{u u}
\end{gathered}
$$




$$
\Delta_{2} u d\left(S_{2} / S_{1}\right) \delta_{2}+\Delta_{1} \delta_{1}=R_{u d}
$$

movimento descendente de $S_{2} / S_{1}$

$$
\begin{gathered}
W_{d u}=\Delta_{2} S_{d u 2} \delta_{2}+\Delta_{1} S_{d u 1} \delta_{1}=\Delta_{2} S_{d u 1} d u\left(S_{2} / S_{1}\right) \delta_{2}+\Delta_{1} S_{d u 1} \delta_{1} \\
W_{d d}=\Delta_{2} S_{d d 2} \delta_{2}+\Delta_{1} S_{d d 1} \delta_{1}=\Delta_{2} S_{d d 1} d d\left(S_{2} / S_{1}\right) \delta_{2}+\Delta_{1} S_{d d 1} \delta_{1} \\
W_{d u}=S_{d u 1}\left\{\Delta_{2} d u\left(S_{2} / S_{1}\right) \delta_{2}+\Delta_{1} \delta_{1}\right\}=S_{d u 1} R_{d u} \\
W_{d d}=S_{d d 1}\left\{\Delta_{2} d d\left(S_{2} / S_{1}\right) \delta_{2}+\Delta_{1} \delta_{1}\right\}=S_{d d 1} R_{d d} \\
\Delta_{2} d u\left(S_{2} / S_{1}\right) \delta_{2}+\Delta_{1} \delta_{1}=R_{d u} \\
\Delta_{2} d d\left(S_{2} / S_{1}\right) \delta_{2}+\Delta_{1} \delta_{1}=R_{d d}
\end{gathered}
$$

Para se encontrar os valores de $\Delta_{1}$ e $\Delta_{2}$ basta resolver ou o sistema de equações simultâneas (13.1) e (13.2) ou (14.1) e (14.2). Logo,

$$
\begin{aligned}
& \Delta_{1}=\left(u R_{u d}-d R_{u v}\right) / \delta_{1}(u-d) \\
& \Delta_{2}=\left(R_{u u}-R_{u d}\right)\left[u(u-d)\left(S_{2} / S_{1}\right) \delta_{2}\right]
\end{aligned}
$$

Considerando o potencial de exercício antecipado (caso das opções americanas) no final do período 1, e se não existem oportunidades de arbitragem sem risco, o valor da opção não pode ser inferior ao valor da carteira equivalente no início do período 2. Se assim fosse, poderia ser obtido um lucro sem risco e sem investimento líquido comprando-se a opção e vendendo-se a carteira. $\mathrm{O}$ contrário não é, necessariamente, verdadeiro, pois o comprador da opção de troca americana que estaria sendo vendida poderia exercê-la imediatamente. Assim, é possível concluir, de acordo com o modelo CRR, que se não existem oportunidades de arbitragem:

\section{movimento ascendente em $S_{2} / S_{1}$ no período 1:}

$$
\begin{aligned}
& W_{u}=\Delta_{2} S_{u 2} \delta_{2}+\Delta_{1} S_{u 1} \delta_{1}, \text { se isto é maior que } S_{u 2}-S_{u 1} \\
& W_{u}=S_{u 2}-S_{u 1}, \text { caso contrário } \\
& \text { Logo, } W_{u}=\max \left(S_{u 2}-S_{u 1}, \Delta_{2} S_{u 2} \delta_{2}+\Delta_{1} S_{u 1} \delta_{1}\right)
\end{aligned}
$$

movimento descendente em $S_{2} / S_{1}$ no período 1:

$$
\begin{aligned}
& W_{d}=\Delta_{2} S_{d 2} \delta_{2}+\Delta_{1} S_{d 1} \delta_{1}, \text { se isto é maior que } S_{d 2}-S_{d 1} \\
& W_{d}=S_{d 2}-S_{d 1}, \text { caso contrário } \\
& \text { Logo, } W_{d}=\max \left(S_{d 2}-S_{d 1}, \Delta_{2} S_{d 2} \delta_{2}+\Delta_{1} S_{d 1} \delta_{1}\right)
\end{aligned}
$$


Substituindo, (15) e (16) em (17) e (18):

$$
\begin{aligned}
& W_{u}=S_{u 1} \max \left\{\left[u\left(S_{2} / S_{1}\right)-1\right],\left[p R_{u u}+(1-p) R_{u d}\right] / \delta_{1}\right\} \\
& W_{d}=S_{d 1} \max \left\{\left[d\left(S_{2} / S_{1}\right)-1\right],\left[p R_{d u}+(1-p) R_{d d}\right] / \delta_{1}\right\}
\end{aligned}
$$

onde,

$$
p \equiv\left(\left(\delta_{1} / \delta_{2}\right)-d\right) /(u-d)
$$

Para apreçar a opção no início do período 1, devemos definir, como antes:

$$
\begin{aligned}
& R_{u}=\max \left\{\left[u\left(S_{2} / S_{1}\right)-1\right],\left[p R_{u u}+(1-p) R_{u d}\right] / \delta_{1}\right\} \\
& R_{d}=\max \left\{\left[d\left(S_{2} / S_{1}\right)-1\right],\left[p R_{d u}+(1-p) R_{d d}\right] / \delta_{1}\right\}
\end{aligned}
$$

tal que $W_{u}=S_{u 1} R_{u}$ e $W_{d}=S_{d 1} R_{d}$.

Portanto,

$$
W=S_{1} \max \left\{\left[\left(S_{2} / S_{1}\right)-1\right],\left[p R_{u}+(1-p) R_{d}\right] / \delta_{1}\right\}
$$

Isto mostra como se pode avaliar uma opção americana de troca binomialmente trabalhandose com backwardation na árvore com preços relativos usando somente o preço corrente de um dos dois ativos no último passo. Em resumo, o apreçamento de uma opção de troca americana por meio de um modelo binomial segue, a partir de uma troca de numerário, os mesmos moldes do modelo original de CRR para uma call vanilla com as seguintes adaptações:

- o preço relativo dos dois ativos substitui o preço do ativo subjacente no problema unidimensional;

- $\delta_{1}$ substitui a taxa de juros;

- $\delta_{2}$ substitui a taxa de dividendos do ativo subjacente;

- 1 é o preço de exercício;

A única resposta que precisa ser dada ainda é como obter os valores de $\mathbf{u}$ e $\mathbf{d}$. Como se pode intuir, estes parâmetros também são obtidos conforme CRR, ou seja:

$$
u=e^{\sigma_{12 \sqrt{\Delta t}}} \quad d=1 / u \quad u d=1
$$

A única diferença reside no termo $\sigma_{12}$, que é o desvio padrão de $\ln \left(S_{2} / S_{1}\right)$, ou seja:

$$
\sigma_{12}^{2}=\sigma_{1}^{2}-2 \sigma_{1} \sigma_{2} \rho_{12}+\sigma_{2}^{2}
$$

Cabe ainda destacar mais dois trabalhos de Rubinstein, ${ }^{14}$ nos quais ele desenvolve um modelo lattice tri-dimensional que faz uso de pirâmides binomiais, as quais nada mais são do que "discretizações" de dois MBGs correlacionados, sendo sua principal vantagem a flexibilidade no trato de opções sobre dois ativos correlacionados.

14 Ver Rubinstein (1994 e 1995). 


\section{EXEMPLOS PRÁTICOS}

Nesta seção usaremos os modelos anteriormente apresentados para apreçar opções do mercado. Para tal, no caso europeu, trabalharemos com o caso simples de uma opção de troca onde utilizaremos a fórmula fechada de Margrabe. Em se tratando de opções americanas, atacaremos o outro problemachave, que é o de avaliação de opção sobre ação com preço de exercício corrigido por variação cambial. Para tal, utilizaremos o modelo binomial de Rubinstein.

\subsection{Caso europeu}

Além da possível criação das spread-lock options para os swaps negociados na BM\&F, em especial os swaps DI-Pré e DI x US\$ comercial, é possível vislumbrar uma aplicação para o caso de operações de troca entre ações ordinárias e preferenciais com ações de emissão de uma mesma empresa, entre ações de empresas do mesmo setor e entre ações de empresas controladas e suas respectivas holdings.

Estes três tipos de operações se traduzem, aqui no Brasil, em uma compra e uma venda simultânea de ações com as características acima, com o intuito de se aproveitar do movimento relativo de preços entre os dois ativos. Assim, a sugestão aqui colocada, de se criar um derivativo que fosse uma opção de troca, traria as seguintes vantagens para a operacionalização destas estratégias:

- Cria-se um instrumento de hedge e alavancagem;

- Sendo seu pay-off pago/recebido por diferença, os custos transacionais seriam minorados, pois não haveria necessidade de troca física de ações, isto é, de compra e vendas de ações, as quais possuem, necessariamente, um valor financeiro maior que o da opção;

- A necessidade de se possuir o ativo a ser vendido ou a obrigação de tomá-lo emprestado deixa de existir, o que, além de ajudar a diminuir custos, facilita a operacionalização;

Abaixo apresentamos uma tabela com os valores das volatilidades e das correlações entre títulos negociados na BOVESPA e que são passíveis se serem objeto de uma opção de troca de ação $\mathrm{ON}$ por $\mathrm{PN}$ e vice-versa.

\section{Tabela 6}

\begin{tabular}{lccc}
\hline & \multicolumn{3}{c}{ Volatilidade } \\
\cline { 2 - 3 } Empresa & ON & PN & Correlação \\
\hline Brasil Telecom Participações & $39,00 \%$ & $35,00 \%$ & $72,00 \%$ \\
Copel & $40,00 \%$ & $48,00 \%$ & $77,00 \%$ \\
Eletrobrás & $52,00 \%$ & $49,00 \%$ & $90,00 \%$ \\
Embraer & $41,00 \%$ & $45,00 \%$ & $83,00 \%$ \\
Embratel & $75,00 \%$ & $70,00 \%$ & $83,00 \%$ \\
Petrobrás & $28,00 \%$ & $26,00 \%$ & $85,00 \%$ \\
Tele Norte Leste Participações & $38,00 \%$ & $34,00 \%$ & $78,00 \%$ \\
Vale do Rio Doce & $30,00 \%$ & $29,00 \%$ & $93,00 \%$ \\
\hline
\end{tabular}

Fonte: Software Economática Versão Agosto-2003.

Período de Cálculo: últimos 252 dias úteis com pregão em 15 de Novembro de 2003. 
Além disto, relacionamos os spreads intra-setoriais e entre empresas do mesmo grupo, também negociados na BOVESPA, conforme abaixo:

Tabela 7

\begin{tabular}{cccc}
\hline & Spread Intra-setorial & \multicolumn{2}{c}{ Spread Holding/Operacional } \\
\hline TNLP4 & BRTP4 & TNLP4 & TMAR5 \\
CPLE6 & CMIG4 & BRTP4 & BRTO4 \\
USIM5 & CSNA3 & GGBR4 & GOAU4 \\
PRGA4 & SDIA4 & ITAU4 & ITSA4 \\
BBDC4 & ITAU4 & & \\
ARCZ6 & VCPA4 & & \\
\hline
\end{tabular}

Daqui podemos depreender o potencial mercado para o derivativo sugerido.

\subsection{Caso americano}

As ações sobre ações com preço de exercício corrigido por variação cambial e pelo IGP-M, negociadas na BOVESPA, são avaliadas, aqui no Brasil, utilizando-se o modelo de Black-Scholes (1973) para opções vanilla européias sobre ações sem dividendos e com preço de exercício determinístico. As únicas adaptações feitas são a utilização da expectativa de variação cambial para o preço de exercício, obtida via utilização da taxa do cupom cambial 'limpo', ${ }^{15}$ para o primeiro caso, e a utilização de uma expectativa de juro real, para o segundo caso, sendo este número obtido por meio da curva de rendimento dos títulos públicos em IGP-M, notadamente as NTN-Cs, ou por meio da estrutura a termo de taxa de juros nominais com o mercado futuro de IGPM.

Cabe aqui ressaltar que para a determinação dos valores de garantias/margens a serem requeridas dos vendedores descobertos destes instrumentos, por parte da Gerência de Controle de Risco da CBLC, não é levada em conta, ou seja, não se embute nenhuma expectativa de risco cambial, ou risco de inflação, e isto tem como conseqüência a existência de restrições, por parte tanto da BOVESPA como da CBLC, para a autorização do lançamento de tais instrumentos, e isto explica, ao menos em parte - ao lado da dificuldade inerente de apreçamento -, a baixa liquidez deste tipo de derivativo.

\subsection{Resultados}

Foram realizados os seguintes exercícios empíricos, conforme detalhado abaixo: ${ }^{16}$

1. Estimamos os valores para opções de troca de ações ordinárias por ações preferenciais para diferentes tempos de maturação e coeficientes de correlação. O objetivo deste teste é verificar se os resultados condizem com a intuição econômica, isto é, prêmios diretamente proporcionais ao tempo de vencimento da opção e inversamente proporcionais ao coeficiente de correlação entre os ativos subjacentes;

15 Esta expectativa é calculada por meio da utilização de interpolações da curva nominal de juros futuros no Brasil, representada pelo DI de um dia, e da curva de cupom cambial 'limpo', obtida dos mercados de forward rate agreement de cupom cambial, ambos negociados na $\mathrm{BM} \& \mathrm{~F}$.

16 No apêndice pode-se encontrar os programas desenvolvidos no MATLAB ${ }^{\circledR}$ para cada um dos exercícios. 
2. Encontramos as diferenças entre os resultados financeiros obtidos em duas estratégias competitivas para aposta em movimentos relativos de preços relativos, ou seja, comparamos os ganhos/ prejuízos obtidos por meio da compra e venda simultânea de ações (da forma como efetivamente se opera tal aposta no Brasil) com os obtidos mediante a compra de uma opção de troca. O objetivo é mostrar que, quando as expectativas com relação ao movimento de preços relativos não se concretizam, ou pior, quando o mercado se move no sentido oposto ao esperado, os prejuízos são minorados por meio da utilização de opções de troca no lugar das compras e vendas simultâneas dos ativos subjacentes;

3. Para o caso americano utilizamos o modelo binomial de Rubinstein (1991a) para uma opção desprotegida de proventos em dinheiro sobre ações preferenciais nominativas da Petrobrás com preço de exercício corrigido por variação cambial e testamos a consistência do modelo, comparando, desta vez, a evolução dos prêmios da opção com o tempo de vida desta e com o número de passos na árvore binomial;

4. Para o mesmo problema acima, verificamos a existência de diferenças significativas entre os prêmios estimados para este tipo de opção utilizando tanto a adaptação do modelo de BlackScholes (1973) para opções européias vanilla sobre ações sem dividendos utilizadas pelo mercado financeiro no Brasil como a abordagem binomial para opções de troca sugerida por Rubinstein (1991a).

As séries usadas são formadas pelos preços de fechamento dos ativos subjacentes nos períodos assinalados apesar de serem estes preços 'viesados' por investidores não tomadores de preços. Poderíamos ter utilizado, no lugar de preços de fechamento, preços médios, caso considerássemos o ponto de vista de um gestor de fundos de investimento, pois a marcação a mercado é feita utilizando preços médios, ou ainda poderíamos ter utilizado preços de pivô ${ }^{17}$ caso considerássemos o ponto de vista de um investidor que baseia suas decisões em análise técnica; além disso, fica óbvio que os valores de pivô, por se tratarem de uma média, contêm mais informação estatística do que cada um de seus valores isolados.

\subsubsection{Prêmios de opções de troca $\times$ tempo de vida $\times$ coeficiente de correlação}

\section{Gráfico 1 - Opção de troca de TNLP3 por TNLP4}

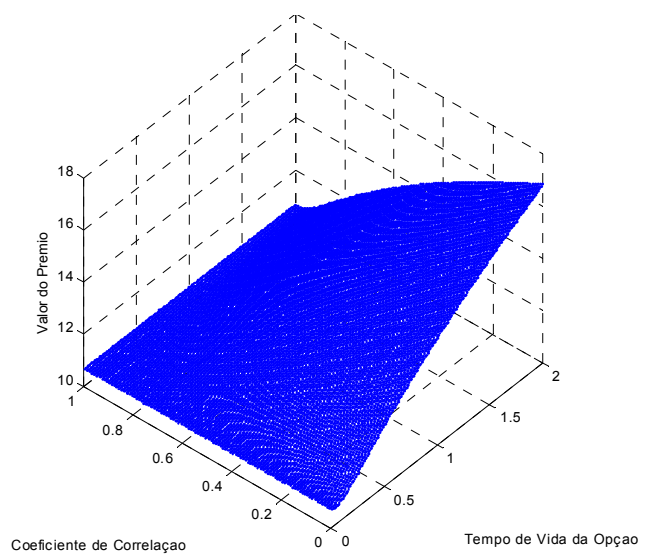

17 É, por definição, uma média aritmética entre os preços máximo, mínimo e médio e fechamento a cada período. 
O gráfico acima confirma nossa intuição, isto é, como podemos observar, os prêmios para uma opção de troca de TNLP3 por TNLP4, calculados segundo a fórmula fechada de Margrabe (1978), são diretamente proporcionais ao tempo de vida da opção e inversamente proporcionais ao coeficiente de correlação entre os dois ativos subjacentes.

\subsubsection{Compra e venda simultâneas dos ativos-objeto $\times$ opção de troca}

Para entendermos melhor o objetivo deste teste, consideremos um investidor que, numa determinada data, queira apostar numa melhor performance das ações preferenciais da Embratel em relação às ações ordinárias da mesma empresa. Ora, para efetivar tal aposta o investidor não possui, no Brasil, nenhum instrumento que permita realizar, com segurança, este tipo de estratégia; efetivamente, ele é obrigado a comprar a ação que teoricamente terá um desempenho melhor e vender a outra (chamemos esta de estratégia 1). Neste ponto, como observamos acima, ou o investidor já possui a ação que irá ser vendida ou deverá alugá-la na CBLC. Isto ocasiona dois problemas: custos e operacionalização. Caso fosse possível negociar uma opção de troca (chamemos esta de estratégia 2) dos dois ativos, os prejuízos, no caso de um movimento adverso de preços, estariam limitados ao prêmio da opção; além disto, a BOVESPA poderia determinar que o pay-off da opção fosse pago por diferença, sem a necessidade de troca física de ações, o que eliminaria o condicionante operacional.

\section{Tabela 8}

\begin{tabular}{ccc}
\hline $\begin{array}{c}\text { Tempo de Vida da Opção } \\
\text { (anos) }\end{array}$ & $\begin{array}{c}\text { Resultado Estratégia 1 - Estratégia 2 } \\
\text { sem Custos Transacionais (R\$) }\end{array}$ & $\begin{array}{c}\text { Resultado Estratégia 1 - Estratégia 2 } \\
\text { com Custos Transacionais (R\$) }\end{array}$ \\
\hline 0.25 & 0.1599 & 0.1278 \\
0.50 & 0.0356 & -0.0060 \\
0.75 & 0.6254 & 0.5845 \\
1.00 & -1.6783 & -1.7395 \\
\hline
\end{tabular}

Estratégia 1: Compra de EBTP4 e Venda de EBTP3.

Estratégia 2: Compra de uma Opção de Troca.

Preços utilizados: Fechamento.

Tempo:0(29/11/2002), 0.25(05/03/2003), 0.50(04/06/2003), 0.75(03/09/2003), 1(28/11/2003).

\subsubsection{Opções sobre ações com preço de exercício corrigido por variação cambial}

O Gráfico 2 mostra a consistência do modelo binomial de Rubinstein para a avaliação de opções sobre ações com preço de exercício corrigido por variação cambial. O modelo, por definição, consegue captar o prêmio extra pela possibilidade de exercício antecipado. Note-se que este prêmio é, como pode ser intuído, diretamente proporcional ao tempo de vida da opção, e em especial no caso das opções desprotegidas para o pagamento de proventos em dinheiro, sejam estes dividendos ou juros sobre o capital próprio. 


\section{Gráfico 2 - Modelo de Rubinstein (1991) - PETR4}

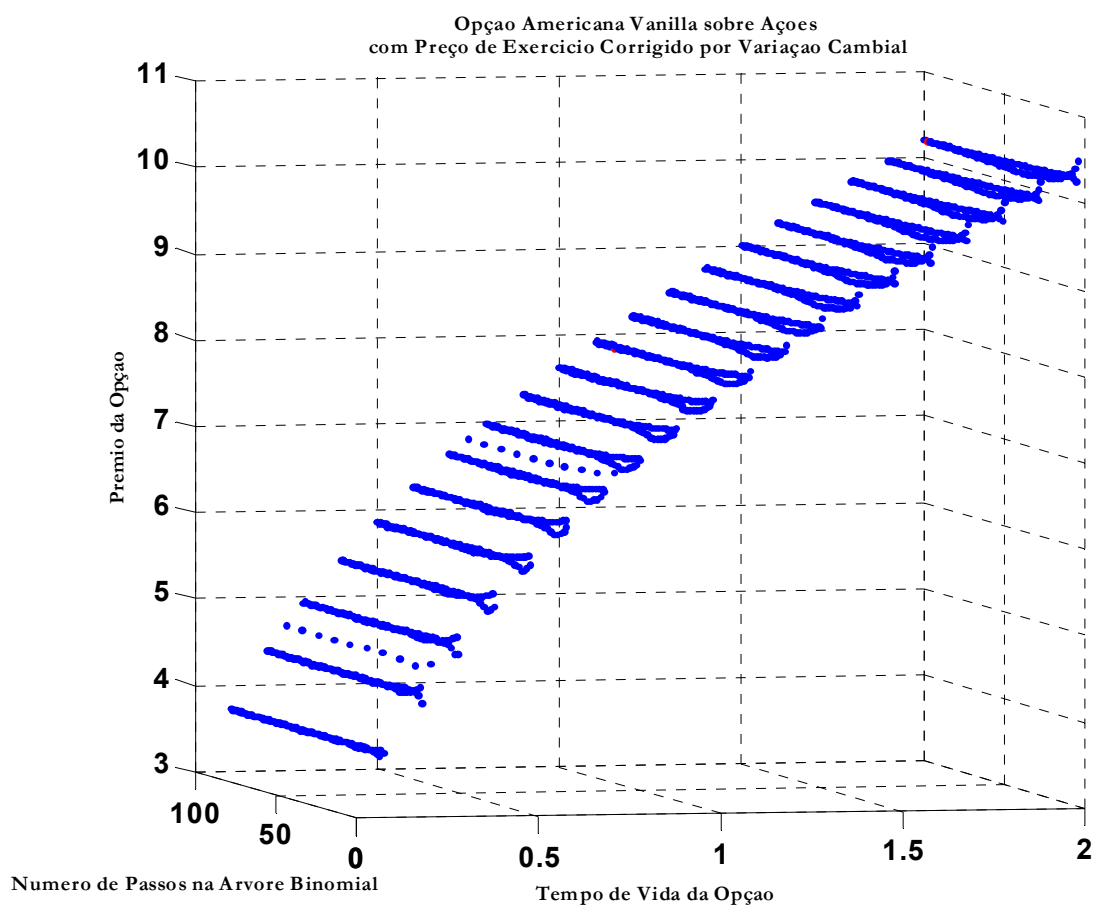

4.3.4 Opções sobre ações com preço de exercício corrigido por variação cambial: Rubinstein (1991) x BlackScholes (1973) adaptado

Os resultados apresentados na Tabela 9 demonstram a existência de divergência entre os prêmios estimados pelos dois modelos, mas não há qualquer indicação que permita escolher um modelo em detrimento do outro.

Como observamos, não existe liquidez neste tipo de derivativo que se traduza em uma série histórica e, portanto, não é possível comparar reais observações de mercado com os prêmios estimados, e mesmo que isto fosse possível, não é estritamente verdadeiro que as reais observações de mercado sejam valores obtidos de forma eficiente, neste caso, sem possibilidades de arbitragem. 
Tabela $9^{18}$

\begin{tabular}{ccccc}
\hline $\begin{array}{c}\text { Tempo de Vida da } \\
\text { Opção (anos) }\end{array}$ & $\begin{array}{c}\text { Black-Scholes (1973) } \\
\text { adaptado }\end{array}$ & $\begin{array}{c}\text { Rubinstein (1991) Opções } \\
\text { de Troca }\end{array}$ & Diferença (R\$) & $\%$ \\
\hline 0.10 & 1,6957 & 2,7698 & $(1,07)$ & $63,34 \%$ \\
0.20 & 2,5407 & 4,1208 & $(1,58)$ & $62,19 \%$ \\
0.30 & 3,1238 & 5,1347 & $(2,01)$ & $64,37 \%$ \\
0.40 & 3,5615 & 5,9748 & $(2,41)$ & $67,76 \%$ \\
0.50 & 3,9029 & 6,7043 & $(2,80)$ & $71,78 \%$ \\
0.60 & 4,1750 & 7,3554 & $(3,18)$ & $76,18 \%$ \\
0.70 & 4,3947 & 7,9473 & $(3,55)$ & $80,84 \%$ \\
0.80 & 4,5734 & 8,4924 & $(3,92)$ & $85,69 \%$ \\
0.90 & 4,7196 & 8,9994 & $(4,28)$ & $90,68 \%$ \\
1.00 & 4,8396 & 9,4746 & $(4,64)$ & $95,77 \%$ \\
\hline
\end{tabular}

Ativo Subjacente: Petrobrás PN (PETR4).

Data de Avaliação: 29/11/2003.

Preço do Ativo Subjacente: preço médio fechamento do dia da avaliação - R\$ 66,60.

Preço de Exercício - R\$ 68,00.

Taxa de Câmbio - PTAX800 do dia da avaliação arredondada para a $2^{\mathrm{a}}$ casa decimal - R\$ 2,95.

Taxa de Juros Nominal: 16\% a.a. (base 252 dias úteis).

FRA de Cupom Cambial: 2\% a.a. (base 360 dias corridos).

Volatilidade do Ativo Objeto: 28\% a.a.

Volatilidade da Taxa de Câmbio: 15\% a.a.

Correlação entre PETR4 e a Taxa de Câmbio: -0,26.

Dividend Yield de PETR4: 5\% a.a.

Taxa de Juros EUA: $1 \%$.

Número de Passos na Árvore: 100.

\section{CONCLUSÕES}

No presente artigo, analisamos os derivativos bidimensionais. Como já mencionado, estes contratos são de muita importância para a cobertura de posições tanto em bolsas de valores como de mercadorias e de futuros, além dos mercados de balcão de vários países, incluindo o brasileiro. Embora neste último o volume de operações não seja significativo, o apreçamento correto destes contratos torna-se imprescindível para a melhor compreensão e uso destes contratos.

O apreçamento de papéis com dois ou mais fatores de risco é um assunto muito interessante e matematicamente complexo. Neste sentido, o modelo de Margrabe (1978) e o modelo de Rubinstein (1991a) mostraram-se de fácil implementação. Como pôde ser observado no caso do apreça-

18 Tanto a taxa de juros nominal em $\mathrm{R} \$$ como a taxa de juros dos EUA e a taxa de cupom cambial são mantidas constantes para os diferentes períodos considerados; porém isto NÂO reflete a realidade. 
mento da opção de troca de TNLP3 por TNLP4 e das opções de PETR4 com preço de exercício corrigido por variação cambial, verificamos que os prêmios são diretamente proporcionais ao tempo de vida da opção e inversamente proporcionais ao coeficiente de correlação entre os dois ativos. No caso da opção de PETR4, o prêmio obtido por meio do modelo de Rubinstein foi maior para todas as maturidades do que o prêmio obtido via Black e Scholes adaptado, o que pode nos dar evidências de que existe um apreçamento errado que conduz a possibilidades de arbitragem. Devido à falta de liquidez nestes contratos, não é possível obter conclusões mais exatas a este respeito.

Fica claro que o apreçamento e uso destes contratos são de muita importância em países como o Brasil, que possuem alto risco cambial. Também foi sugerido que outros fatores de correção poderiam ser considerados pela $\mathrm{BM} \& \mathrm{~F}$, tais como a inflação, o que permitiria um melhor hedging.

Por outra parte, como já mencionado, existem modelos que consideram processos mais sofisticados e que requerem maior esforço computacional, porém mais realistas, no sentido de que capturam melhor o comportamento dos retornos dos ativos subjacentes, como, por exemplo, os considerados por Gerber e Shiu (1996) e Fajardo e Mordecki (2003), nos quais é permitida a presença de caudas grossas.

Um problema não analisado neste artigo é o relacionado aos mercados incompletos e à existência de diferentes medidas martingalas equivalentes. O leitor interessado neste tipo de problema pode dispor de uma metodologia, que permite encontrar estas medidas, em Fajardo (2004). Infelizmente, a implementação requer uma base de dados substancial que permita a estimação dos parâmetros, e que não existe atualmente no Brasil, dado o baixo volume de operações.

\section{APÊNDICE: CóDIGOS EM MATLAB®}

\section{Apreçamento de opção de troca utilizando a fórmula de Margrabe}

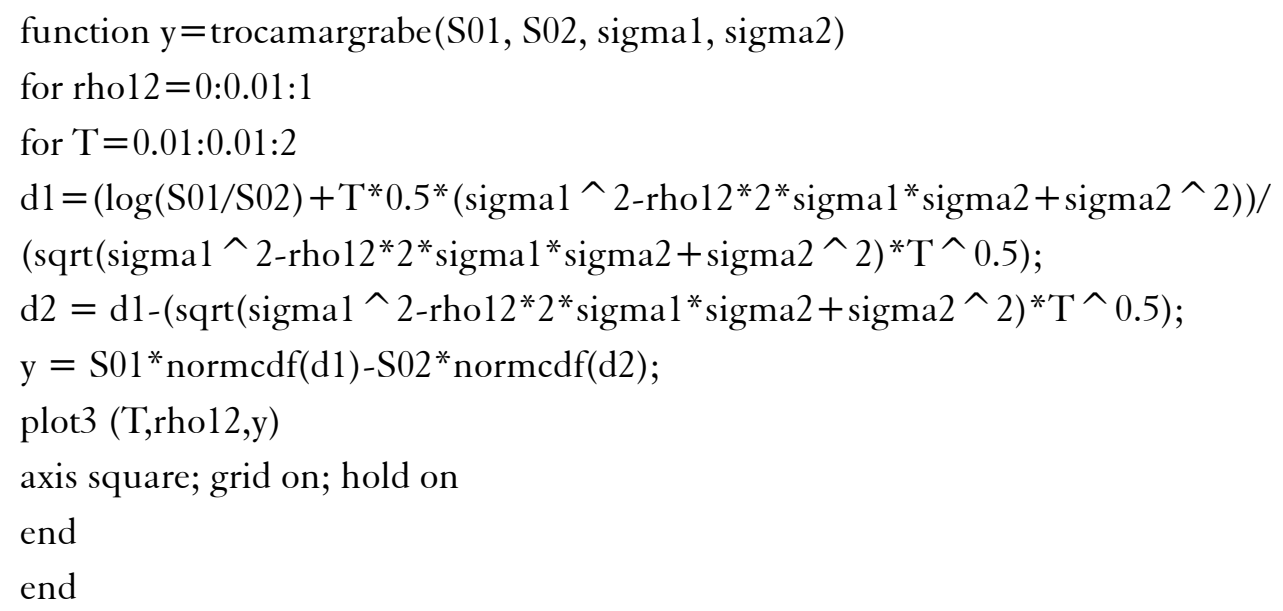

Apreçamento de opção vanilla americana com preço de exercício corrigido por variação cambial com o modelo de Black-Scholes (1973) adaptado

\% Código para Avaliação de Opção Americana Vanilla com Preço de Exercício em Dólar $\%$ não protegida para proventos em dinheiro com o modelo de Black-Scholes (1973) adaptado 
function y $=$ kdolar(S0, K, sigma, e, tipo, DU, DC, R, FRA)

$\% \mathrm{~S}$ é o preço do ativo subjacente e $\mathrm{K}$ e o preço de exercício em $\mathrm{R} \$$

$\%$ sigma é a volatilidade do ativo subjacente

$\%$ é a taxa de câmbio spot

$\%$ tipo $=1$ ou 0 , para call ou put, respectivamente

\% DU número de dias úteis e DC número de dias corridos até o vencimento da opção

$\%$ R juro nominal, interpolado da curva do DI futuro na $\mathrm{BM} \& \mathrm{~F}$, para o vencimento da opção

$\%$ FRA taxa do forward rate agreement de cupom cambial para o vencimento da opção

\% Cálculo da Expectativa de Variação Cambial

$\mathrm{Ee}=\left((1+\mathrm{R}) \wedge(\mathrm{DU} / 252) /\left(1+\mathrm{FRA}^{*} \mathrm{DC} / 360\right)\right)-1$;

$\%$ Cálculo da Expectativa do Dólar Futuro

\% Cálculo do Prêmio da Opção

$\mathrm{d} 1=\left(\log \left(\mathrm{S} 0 / \mathrm{K}^{*}(1+\mathrm{Ee})\right)+(\log (1+\mathrm{R})+\operatorname{sigma} \wedge 2 / 2)^{*}(\mathrm{DU} / 252)\right) /\left(\operatorname{sqrt}\left(\operatorname{sigma} \wedge 2^{*}(\mathrm{DU} / 252)\right)\right) ;$

$\mathrm{d} 2=\mathrm{d} 1-(\operatorname{sqrt}(\operatorname{sigma} \wedge 2 *(\mathrm{DU} / 252)))$;

if tipo $==1$

$\mathrm{Nd} 1=\operatorname{normcdf}(\mathrm{d} 1)$;

$\mathrm{Nd} 2=\operatorname{normcdf}(\mathrm{d} 2)$;

else

$\mathrm{Nd} 1=\operatorname{normcdf}(-\mathrm{d} 1)$;

$\mathrm{Nd} 2=\operatorname{normcdf}(-\mathrm{d} 2)$;

end

if tipo $==1$

kdolar $=\mathrm{S} 0 * \mathrm{Nd} 1-\mathrm{K}^{*}(1+\mathrm{Ee}){ }^{*} \exp (-\log (1+\mathrm{R}) *(\mathrm{DU} / 252)) * \mathrm{Nd} 2$

else

kdolar $=-\mathrm{S}^{*} \mathrm{Nd} 1+\mathrm{K}^{*}(1+\mathrm{Ee}){ }^{*} \exp (-\log (1+\mathrm{R}) *(\mathrm{DU} / 252))^{*} \mathrm{Nd} 2$

end

Estratégia de compra e venda simultânea de ações versus opção de troca

\% Estratégia Principal (BOVESPA): comprar ação PN e vender ação ON

$\%$ Estratégia Sugerida: comprar uma Opção de Troca

\% 1 - São 4 os horizontes de Tempo: 12, 9, 6 e 3 meses até o vencimento da opção

$\% 2$ - Série Histórica de Preços: 1 ano de preços de fechamento diários das duas ações

$\% 3$ - Custo de Oportunidade é desconsiderado

$\% 4$ - Troca de Ações propriamente dita é desfeita no dia do exercício da opção

$\% 5$ - De posse da série histórica de preços calcula-se a matriz VA de variâncias-covariâncias

$\%$ (abaixo imputamos diretamente sigma1, sigma2 e cov12)

function $\mathrm{y}=\operatorname{troca}(\mathrm{S} 01, \mathrm{~S} 02$, sigma1, sigma2, sigma12)

$\%$ Custos de Transação (em \%) 
emolumento $=0.00035$

registro $=0.001$

corretagem $=0.005$

devolução $=0.7$

$\%$ Para calcular os valores sem considerar os custos igualamos os valores acima a zero

rho12 $=\operatorname{sigma12} \uparrow 2 /($ sigma $1 *$ sigma 2$)$

sigmaquadrado $=\left(\operatorname{sigma1} \wedge 2-\mathrm{rhol}_{2} * 2 * \operatorname{sigma} 1 * \operatorname{sigma} 2+\operatorname{sigma} 2 \wedge 2\right)$

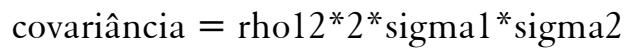

\% Agora usamos o Código para Opção de Troca pela Fórmula de Margrabe

$\mathrm{T}=$ linspace $(0.25,1,4)$

$\mathrm{d} 1=\left(\log (\mathrm{S} 01 / \mathrm{S} 02)+\mathrm{T} .{ }^{*} 0.5^{*}(\operatorname{sigmaquadrado})\right) /\left(\operatorname{sqrt}(\operatorname{sigmaquadrado}){ }^{*} \mathrm{~T} . \wedge 0.5\right)$

$\mathrm{d} 2=\mathrm{d} 1$-sqrt(T.* ${ }^{*}$ sigmaquadrado)

trocamargrabe $=(-(\mathrm{S} 01 * \text { normcdf }(\mathrm{d} 1)-\mathrm{S} 02 * \text { normcdf }(\mathrm{d} 2)))^{\prime *}(1+($ emolumento + registro + corretagem* ${ }^{*}(1-$ devolução) $\left.)\right)$

$\%$ Cálculo do desembolso/recebimento de caixa inicial

$\mathrm{p} 1=-\mathrm{S} 01 *\left(1+\left(\right.\right.$ emolumento + registro + corretagem $^{*}(1$-devolução $\left.\left.)\right)\right)$

$\mathrm{p} 2=\mathrm{S} 02 *(1-($ emolumento + registro + corretagem $*(1$-devolução $)))$

$\mathrm{cS} 1 \mathrm{vS} 2=\mathrm{p} 1+\mathrm{p} 2$

\% Cálculo do desembolso/recebimento de caixa final (reversão da operação)

$\%$ Precisamos, em primeiro lugar, da matriz de preços de fechamento das duas ações

$\%$ a cada data de vencimento S1S2final

S1S2final $=[($ imputar aqui os valores de S1 e S2 de fechamento a cada T) $]$

$\mathrm{i}=$ linspace $(1,4,4)$;

vS 1 c S2 $=+$ S 1 S 2 final $(i, 1) *(1-($ e molumento + corretagem* $(1-$ devolução $)))-$

S1S2final $(i, 2) *\left(1+\left(\right.\right.$ emolumento + corretagem* ${ }^{*}(1-$ devolução $\left.\left.)\right)\right)$

\% Cálculo do Pay-off da Opção

Pay-off $(\mathrm{i}, 1)=\max (0,+\mathrm{S} 1 \mathrm{~S} 2$ final $(\mathrm{i}, 1) *(1-($ emolumento + registro + corretagem * $(1-$ devolução $)))-$ S1S2final $(i, 2) *\left(1-\right.$ emolumento + registro + corretagem $^{*}(1-$ devolução $\left.\left.)\right)\right)$

$\%$ Resultado Final da Operação de Troca de Papéis

resultadoS1S2 $=\mathrm{vS} 1 \mathrm{cS} 2+\mathrm{cS} 1 \mathrm{vS} 2$

$\%$ Resultado Final da Compra da Opção

Resultado da opção = pay-off + trocamargrabe

$\%$ L/P da Estratégia Efetiva Troca de Ações em vez de Compra da Opção de Troca

L_Ptrocanormal $=$-resultadoopção + resultadoS1S2

plot(T,L_Ptrocanormal,'--rs','LineWidth',2,'MarkerEdgeColor','k','MarkerFaceColor','g', 'MarkerSi$\left.\mathrm{ze}^{\prime}, 10\right)$

\section{Apreçamento de opção de troca com a abordagem binomial de Rubinstein}

\% Código para Avaliação de Opção Americana Vanilla com Preço de Exercício em Dólar 
\% não protegida para proventos em dinheiro como uma Opção de Troca

$\%$ Modelo de Mark Rubinstein "One for Another", RISK (July 1991)

$\% \mathrm{~K}=$ preço de exercício, inicial, em pontos

$\%$ e $=$ taxa de câmbio $(\mathrm{R} \$ / \mathrm{US} \$)$

$\%$ delta 2 = taxa contínua de pagamento do ativo 2 a cada período (dividend yield)

$\%$ delta 1 = taxa contínua de pagamento do "ativo $1 "$ a cada período (taxa de juros estrangeira)

$\%$ sigma2 $=$ volatilidade do ativo 2 (ação)

$\%$ sigma1 = volatilidade do "ativo 1" (taxa de câmbio)

$\%$ rho $=$ correlação entre o ativo 1 e 2

$\% \mathrm{~T}=$ tempo para o vencimento

$\% \mathrm{~N}=$ número de passos na árvore

\% Pay-off da Opção: $\max (\mathrm{S} 02$ - Ke;0)

function y=trocabinomial(S02,K,e,delta2,delta1,sigma2,sigma1,rho,T,N)

$\%$ Tamanho do Passo no Tempo e Volatilidade

$\mathrm{dt}=\mathrm{T} / \mathrm{N}$

$\operatorname{sigma}=\operatorname{sqrt}\left(\operatorname{sigma} 1 \wedge 2+\operatorname{sigma} 2 \wedge 2-2 *\right.$ rho $\left.^{*} \operatorname{sigma} 1 * \operatorname{sigma} 2\right)$

$\%$ Cálculo da Probabilidade de um Movimento Ascendente e de um Movimento Descendente

$\mathrm{u}=\exp \left(\operatorname{sigma}{ }^{*} \operatorname{sqrt}(\mathrm{dt})\right)$

$\mathrm{d}=1 / \mathrm{u}$

$\mathrm{p}=(\exp (($ delta $1-$ delta 2$) * \mathrm{dt})-\mathrm{d}) /(\mathrm{u}-\mathrm{d})$

$\%$ Constrói a Árvore de Preços do Ativo seguindo um Processo Binomial

for $i=1: N+1$

for $\mathrm{j}=\mathrm{i}: \mathrm{N}+1$

Stree $(\mathrm{i}, \mathrm{j})=\left(\mathrm{S} 02 /\left(\mathrm{K}^{*} \mathrm{e}\right)\right)^{*}\left(\mathrm{~d}^{\wedge}(\mathrm{i}-1)\right)^{*}(\mathrm{u} \wedge(\mathrm{j}-\mathrm{i}))$

end

end

$\%$ Constrói Árvore de Pay-offs da Opção seguindo um Processo Binomial

for $\mathrm{i}=1: \mathrm{N}+1$

for $j=i: N+1$

Streefinal $(i, j)=\operatorname{Stree}(i, j)-1$

end

end

\% Condição de Contorno da Opção

for $\mathrm{i}=1: \mathrm{N}+1$

if Stree $(\mathrm{i}, \mathrm{N}+1)>1$

ftree $(\mathrm{i}, \mathrm{N}+1)=$ Stree $(\mathrm{i}, \mathrm{N}+1)-1$

else ftree $(\mathrm{i}, \mathrm{N}+1)=0$

end

end 
\% Como a opção e americana, testa-se para checar se seu valor intrínseco e maior do que o seu preço \% esperado. Se for, ela é exercida.

for $\mathrm{j}=\mathrm{N}:-1: 1$

for $\mathrm{i}=1: \mathrm{j}$

ftree $(i, j)=\max \left(\exp \left(-(\text { delta1-delta } 2)^{*} \mathrm{dt}\right) *\left(\mathrm{p}^{*}\right.\right.$ ftree $(\mathrm{i}, \mathrm{j}+1)+(1-\mathrm{p})^{*}$ ftree $\left.(i+1, j+1)\right)$, Stree $\left.(i, j)-1\right)$

end

end

binomial $=\mathrm{K}^{*} \mathrm{e}^{*}$ ftree $(1,1)$

\section{REFERÊNCIAS}

Black, F.; Scholes, M. The pricing of options and corporate liabilities. Journal of Political Economy, v. 81, 1973.

Boyle, P. Options: a Monte Carlo approach. Journal of Financial Economics, v. 4, p. 323-338, 1977.

. A lattice framework for option pricing with two state variables. Journal of Financial and Quantitative Analysis, n. 1, p. 1-12, 1988.

Boyle, P.; Evnine, J.; Gibbs, S. Numerical evaluation of multivariate contingent claims. Review of Financial Studies, n. 2, 1889.

Boyle, P.; Tse, Y. K. An algorithm for computing values of options on the maximum or minimum of several assets. Journal of Financial and Quantitative Analysis, v. 25, n. 2, p. 215-227, 1990.

Cox, J. C.; Ross, S. A. The valuation of options for alternative stochastic processes. Journal of Financial Economics, n. 7, p. 145-166, 1976.

Cox, J. C.; Ross, S. A.; Rubinstein, M. Option pricing: a simplified approach. Journal of Financial Economics, n. 7, p. 229-263, 1979.

Fajardo, J. S. Equivalent Martingale measures and Lévy processes. IBMEC Working Paper, 2004.

Fajardo, J. S.; Mordecki, E. Pricing derivatives on two Lévy-Driven stocks. Proceedings of the Seven Annual Meeting on Insurance: Mathematics and Economics. Lyon, France, 2003.

Gerber, H. U.; Shiu, E. S. W. Option pricing by Esscher-transform. Transactions of the Society of Actuaries, v. 46, p. 99-191, 1994.

. Martingale approach to pricing perpetual american options on two stocks. Mathematical Finance, v. 6, n. 3, p. 303-322, 1996.

Harrison, J. M.; Pliska, S. R. Martingales and stochastic integrals in the theory of continuous trading. Stochastic Processes and Their Applications, 11, p. 215-260, 1981.

Johnson, H. Options on the maximum or the minimum of several assets. Journal of Financial and Quantitative Analysis, v. 22, p. 277-283, 1987.

Kamrad, B.; Ritchken, P. Multinomial approximating models for option with K state variables. Management Science, v. 37, n. 12, 1991.

Margrabe, W. The value of an option to exchange one asset for another. The Journal of Finance, 33, p. 177-186, 1978.

. Triangular equilibrium and arbitrage in the market for options to exchange one asset for another. Journal of Derivatives, 1, p. 60-69, 1993.

Merton, R. C. The theory of rational option pricing. The Bell Journal of Economics and Management Science, v. 4, 1973.

Rubinstein, M. Exchange options. UC Berkeley, 1991a. Não publicado. 
. One for another. Risk, v. 4, n. 7, p. 30-32, 1991 b.

Options for the undecided. Risk Magazine, 4, 1991c.

. Somewhere over the rainbow. Risk, n. 10, p. 63-66, 1991d.

. Return to OZ. Risk, 7, p. 67-71, 1994.

. Rainbow options. 1995.

. On the relation between binomial and trinomial option pricing models. Journal of Derivatives, 2000.

Stulz, R. Options on the minimum or the maximum of two risky assets: analysis and applications. Journal of Financial Economics, 10, p. 161-185, 1982. 